“C 2010 IEEE. Personal use of this material is permitted. Permission from IEEE must be obtained for all other uses, in any current or future media, including reprinting/republishing this material for advertising or promotional purposes, creating new collective works, for resale or redistribution to servers or lists, or reuse of any copyrighted component of this work in other works.” 


\title{
Simulation of PV Array Characteristics and Fabrication of Microcontroller Based MPPT
}

\author{
Md. Rabiul Islam ${ }^{\# 1}$, Youguang Guo", Jian Guo Zhu\#, M. G. Rabbani” \\ "Faculty of Engineering and Information Technology, University of Technology Sydney, Australia \\ "Rajshahi University of Engineering \& Technology, Rajshahi-6204, Bangladesh \\ 1E-mail: rabiulbd@ hotmail.com,mdrabiul.islam@student.uts.edu.au
}

\begin{abstract}
In photovoltaic (PV) systems, the PV array represents about $57 \%$ of the total cost of the system, so it is very desirable to operate the $\mathrm{PV}$ panel at the maximum power point (MPP). The output characteristics of the solar array are nonlinear and vitally affected by solar radiation, temperature, and load conditions. In this respect simulation analysis of PV array is essential to understand the output characteristics of $P V$ array such that it can operate at the maximum power producing point for a given atmospheric condition. This paper explores the output characteristics of a PV array in MATLAB environment and the fabrication of a microcontroller based simple maximum power point tracker (MPPT). The fabricated MMPT has the capability to track the MPP and the use of the MPPT enhances the output power by $20 \%$.
\end{abstract}

Keywords - PV array, characteristics simulation, microcontroller, MPPT, fabrication

\section{INTRODUCTION}

Photovoltaic (PV) generation is getting increasing importance as a renewable source due to the advantages such as the absence of fuel cost, little maintenance and no noise and wear due to absence of moving parts. However, two important factors limit the implementation of photovoltaic systems, i.e. high cost and low efficiency in energy conversion. It was mentioned that the conversion efficiency of the solar PV module is quite low at only $10-17 \%$ [1]. In photovoltaic systems, the PV array represents about $57 \%$ of the total cost of the system, and the battery storage system corresponds to $30 \%$ of the cost. Other system components such as inverters and maximum power point (MPP) tracker contribute to only $7 \%$ of the cost [2]. Due to the low conversion efficiency and high cost of solar array, it is very desirable to operate the PV panel at the MPP. The output characteristics of the solar array are nonlinear and critically affected by solar radiation, temperature, and load conditions. This paper presents the output characteristics of a PV array for various atmospheric conditions. Maximization of power output improves the utilization of the solar PV module. An MMP tracker (MPPT) is used for extracting the maximum power from the solar PV module and transferring power to the load. According to the maximum power transfer theory, the power delivered to the load is the maximum when the source internal impedance matches the load impedance. A DC-DC converter (step up/step down) can be used to serve the purpose of transferring maximum power from the solar PV module to the load. A DC-DC converter acts as an interface between the load and the solar PV module. An MPPT is an electronic system that varies the electrical operating point of the modules so that they are able to deliver the maximum power. It can be used with a mechanical tracking system. Without an MPPT the solar modules are connected directly to the load. This forces the modules to operate at the load voltage, which is usually not the optimal operating voltage at which the modules are able to produce their maximum power. By changing the duty cycle the load resistance as seen by the source can be varied and matched at the point of the peak power with the source so as to transfer the maximum power. An MPPT is required to meet the goals, e.g., to operate closely to MPP, provide high conversion efficiency and maintain tracking for a wide range of variation in environmental conditions [3]. In this paper a microcontroller based simple MPPT is also fabricated and tested in the laboratory.

\section{PV Cell Equivalent Circuit}

An ideal solar cell may be modeled by a current source in parallel with a diode. In practice no solar cell is ideal and hence a shunt resistance and a series resistance are added to the model as shown in Fig. 1. $R_{\text {scell }}$ is the intrinsic series resistance of the solar cell whose value is usually very small. $R_{\text {shcell }}$ is the equivalent shunt resistance of the solar cell whose value is usually very large.

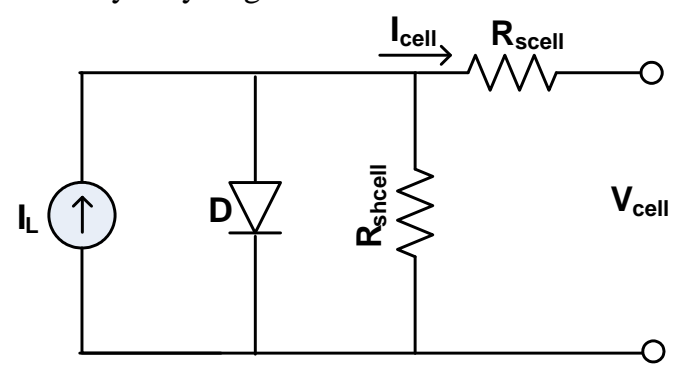

Fig. 1 Equivalent circuit of PV cell

The PV arrays are built up with series and/or parallel connected combinations of solar cells. Therefore, for an array with $n_{S} \times n_{P}$ cells the current equation [4]-[6] is

$I_{P V}=n_{p} I_{L}-n_{p} I_{o}\left[\exp \left(G\left(\frac{V_{P V}+I_{P V} R_{s}}{n_{s}}\right)\right)-1\right]-\frac{V_{P V}+I_{P V} R_{s}}{R_{s h}}$

where $I_{P V}=n_{P} I_{\text {cell }}$ is the $\mathrm{PV}$ array output current, $V_{P V}=n_{S} V_{\text {cell }}$ is the $\mathrm{PV}$ array output voltage, $\mathrm{n}_{\mathrm{s}}$ is the number of cells connected in series, $\mathrm{n}_{\mathrm{p}}$ is the number of panels connected in parallel, $R_{S}=R_{\text {Scell }} \frac{n_{S}}{n_{P}}$ is the PV array series resistance, $R_{s h}=R_{\text {shcell }} \frac{n_{S}}{n_{P}}$ is the PV array shunt resistance, $I_{\text {cell }}$ is the cell output current, $I_{L}$ is the light-generated current, and $I_{0}$ is the cell saturation current. 
The shunt resistance $R_{\text {shcell }}$ is much greater than the series resistance $R_{\text {scell }}$. The simplified cell model is shown in Fig. 2 .

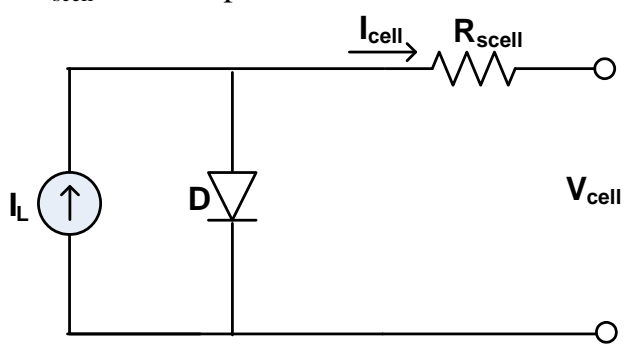

Fig. 2 Simplified equivalent circuit of PV cell

Therefore, the array current-voltage relation becomes

$$
I_{P V}=n_{p} I_{L}-n_{p} I_{o}\left[\exp \left(G\left(\frac{V_{P V}+I_{P V} R_{s}}{n_{s}}\right)\right)-1\right]
$$

\section{PV ARRAY CHARACTERISTIC}

The current-to-voltage characteristic of a solar cell is nonlinear, which makes it difficult to determine the MPP. Figs. 3 and 4 illustrates the simulated $\mathrm{I}-\mathrm{V}$ and $\mathrm{P}-\mathrm{V}$ characteristic of the solar array under a fixed temperature $\left(25^{\circ} \mathrm{C}\right)$ and a fixed irradiance of $1000 \mathrm{~W} / \mathrm{m}^{2}$, respectively. The I-V and P-V characteristics for various irradiance at fixed temperature $\left(25^{\circ} \mathrm{C}\right.$ ) are shown in Figs. 5 and 6, respectively. Fig. 3 illustrates the operating characteristic curves of the solar array under a given insolation. It consists of two regions: one is the current source region, and the other is the voltage source region. In the voltage source region (on the right side of the curve), the internal impedance of the solar array is low, and in the current source region (on the left side of the curve), the internal impedance of the solar array is high. The MPP of the solar array is located at the knee of the curve.

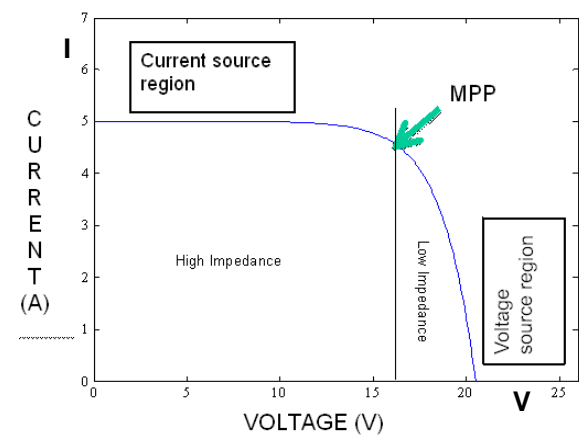

Fig. 3 I-V characteristic of the solar array

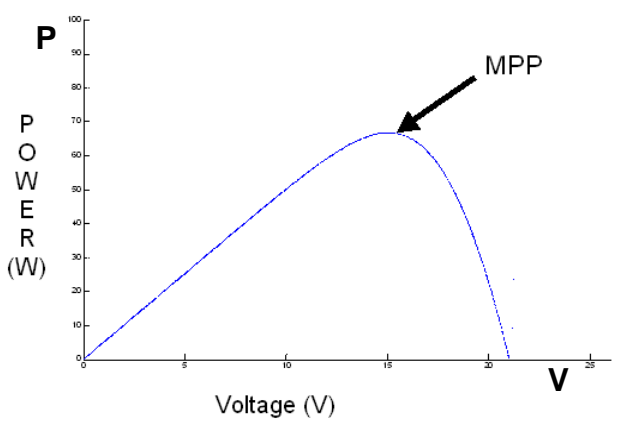

Fig. 4 P-V characteristic of the solar array

Besides irradiance, temperature also plays an important role in predicting the $\mathrm{I}-\mathrm{V}$ characteristic, and the effects of both factors have to be considered when designing a PV system. Whereas the irradiance mainly affects the output current, the temperature mainly affects the terminal voltage. Figs. 7 and 8 show the simulated I-V and P-V characteristic for various temperatures at fixed irradiance of $1000 \mathrm{~W} / \mathrm{m}^{2}$, respectively.

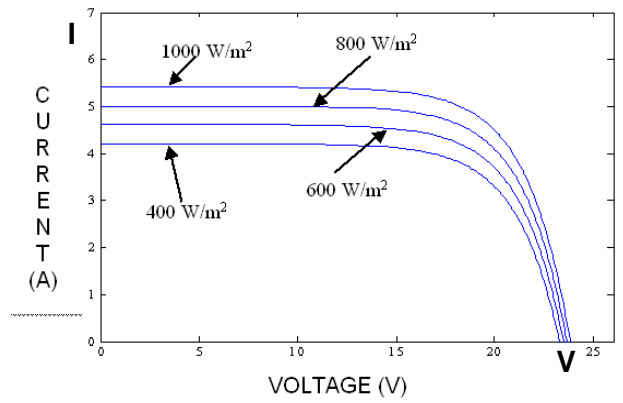

Fig. 5 I-V characteristic of the solar array under a fixed temperature but different irradiance

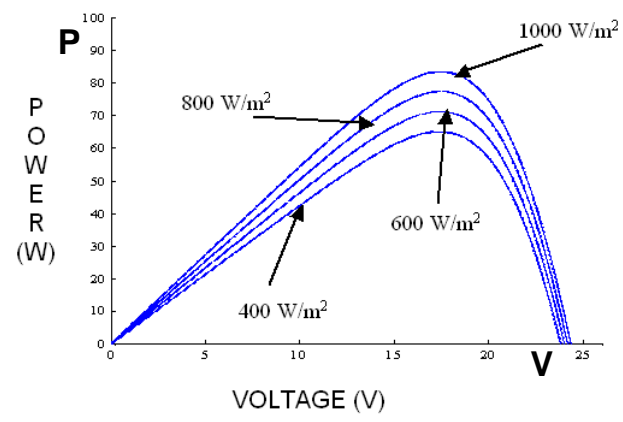

Fig. 6 P-V characteristic of the solar array under a fixed temperature but different irradiance

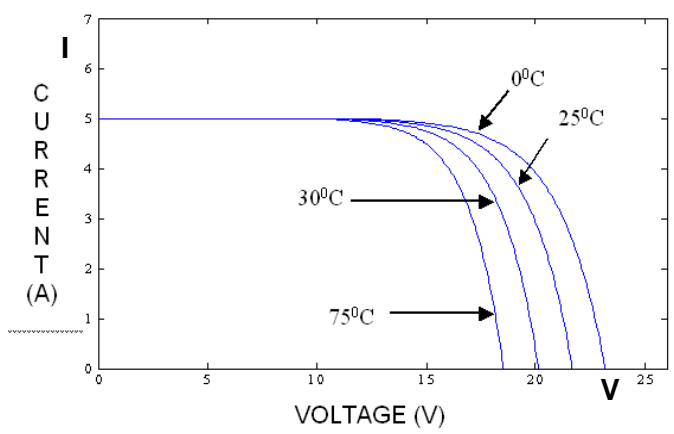

Fig. 7 I-V characteristic of the solar array under a fixed irradiance but different temperature

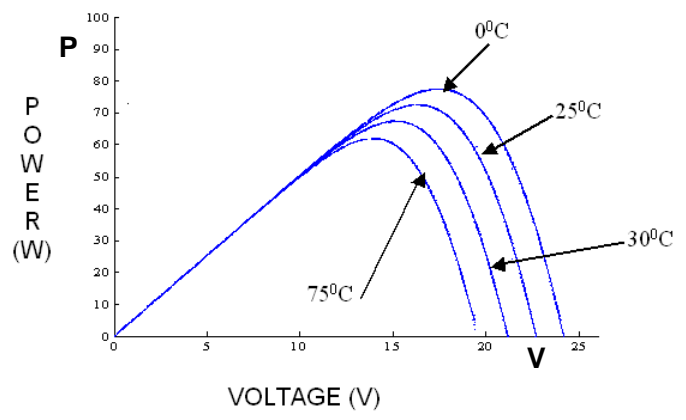

Fig. 8 P-V characteristic of the solar array under a fixed irradiance but different temperature

\section{IMPEDANCE MATCHING MPPT}

Fig. 9 shows the block diagram of the MPPT. The main component of the MPPT is the boost converter that steps up the solar panel output voltage to the desired load voltage. To 
ensure that the solar module operates at the MPP, the input impedance of the converter must be adapted to force the solar module to work at its MPP.

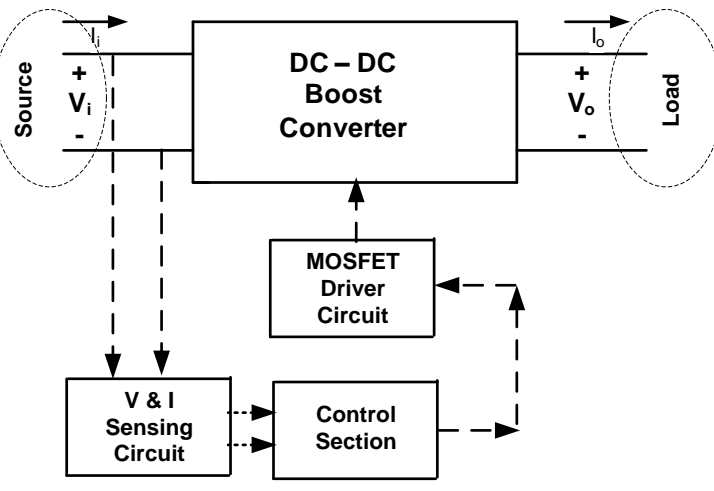

Fig. 9 Block diagram of MPPT

According to the maximum power transfer theory, the power delivered to the load is maximum when the source internal impedance matches the load impedance. A DC-DC converter can be used to serve the purpose of transferring maximum power from the solar PV module to the load. A DC-DC converter acts as an interface between the load and the solar PV module. If the system is required to operate at or near the MPPs of the solar array, the impedance seen from the converter input side (can be adjusted by PWM control signal) needs to match the internal impedance of the solar array. By changing the duty cycle the load impedance as seen by the source is varied and matched at the point of the peak power with the source so as to transfer the maximum power. The relation between duty cycle, $\mathrm{D}$ and impedance seen from converter input side is as

$$
R_{\text {equiv }}=(1-D)^{2} \times R_{L}
$$

The ATmega32 microcontroller operating at a speed of $8 \mathrm{MHz}$ is used to carry out the algorithm. The program flow chart for this algorithm is shown in Fig. 10. The algorithm reads the value of current and voltage from the solar PV module. Power is then calculated from the measured voltage and current.

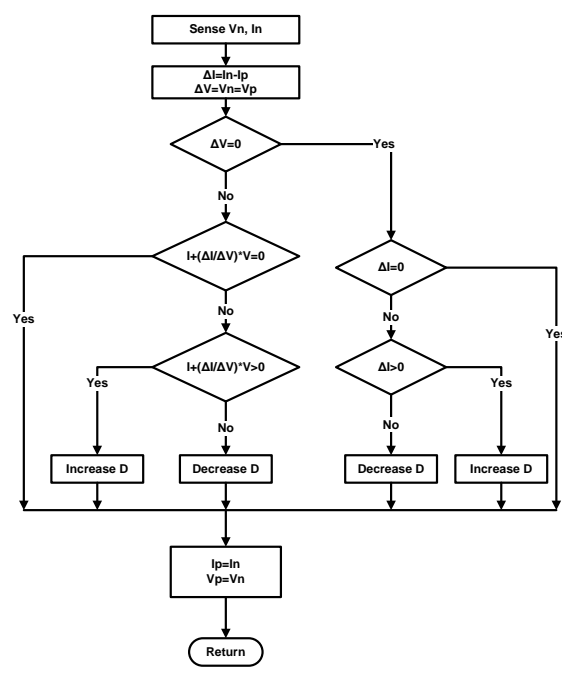

Fig. 10 Flowchart of the tracking algorithm

The value of voltage and power at the $\mathrm{k}^{\text {th }}$ instant are stored. Then next values at the $(\mathrm{k}+1)^{\text {th }}$ instant are measured again and power is calculated from the measured values. The power and voltage at the $(\mathrm{k}+1)^{\text {th }}$ instant are subtracted with the values from the $\mathrm{k}^{\text {th }}$ instant. It is observed from the power voltage curve of the solar PV module that in the right hand side curve where the voltage is almost constant the slope of power voltage is negative $(\mathrm{dP} / \mathrm{dV}<0)$, whereas in the left hand side the slope is positive $(\mathrm{dP} / \mathrm{dV}>0)$. The right side curve is for the lower duty cycle (near zero) whereas the left side curve is for the higher duty cycle (near unity). The program then returns and starts tracking again until the MPP is reached. The tracking process duration is 1 minute. But still, if the sample time is reduced in direct duty cycle control the power tracking can be made faster even in the fast varying climatic conditions. The maximum duty cycle ratio is set at $90 \%$ and the minimum is at $10 \%$ to avoid power loss.

\section{PROTOTYPE OF MPPT}

Microcontroller is used for the control of the MPPT system. The selected algorithm is written in $\mathrm{C}$ and then is compiled by a freeware version C compiler "WinAVR". Then the program is burnt into the microcontroller memory. The controller acquires the necessary data from the solar PV module (panel voltage and current) and sends the control signals to the algorithm which works on the signals and gives necessary PWM signals to drive the MOSFET.

Fig. 11 shows the hardware setup of the MPPT system. The experiment was performed in both indoor and outdoor environments. The outdoor setup involved taking measurements under bright sunshine, slightly cloudy and very cloudy conditions. This allowed for the observation of the solar module's response to varying irradiance levels. The indoor setup involved taking measurements under two artificial lighting conditions. The artificial lighting was achieved by using the laboratory's fluorescent lighting and an incandescent lamp. The different ambient temperature between the outdoor setup and the indoor setup allows for the observation of the module's response to varying temperature conditions.

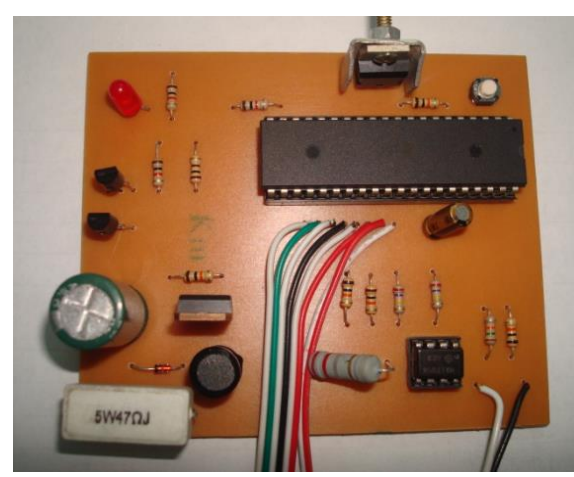

Fig. 11 Photograph of MPPT (PCB)

\section{RESULTS AND DISCUSSION}

The output voltage reaches the highest value under bright sunshine and decreases under very cloudy conditions to less than half the output under bright conditions. The effect of partial shadow on the output voltage is also investigated and it is observed that a partial shadow on any part of the solar module will lead to a reduction of the output voltage.

The experiment was also performed under indoor conditions where the ambient temperature is $25{ }^{\circ} \mathrm{C}$, the same temperature as the manufacturer's test conditions. The indoor illumination was significantly less than the outdoor illumination. But the output voltage in both indoor illumination conditions is higher than the output voltage under very cloudy outdoor conditions. The much lower 
indoor module temperature may be responsible for this and it demonstrates that higher irradiation levels do not necessarily lead to a higher output voltage because of the accompanying increase in temperature.

The PWM signal is generated through the microcontroller. The microcontroller generates signals with frequency up to 8 $\mathrm{MHz}$. In this case the switching frequency has been considered to be $33 \mathrm{kHz}$. Fig. 12 shows the PWM output signal from the microcontroller.

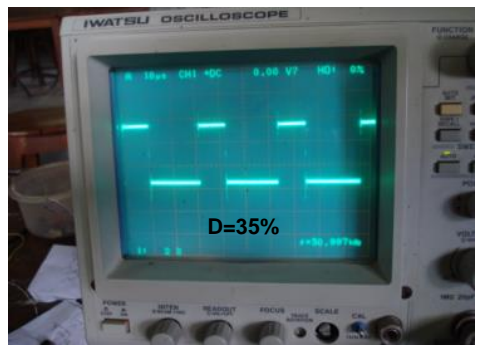

Fig. 12 Photograph of microcontroller's output PWM signal

Fig. 13 shows the inductor current wave shape (voltage across $1 \Omega$ resistor placed in series with the inductor). It has been observed that the converter works in continuous conduction mode. The output voltage of the boost converter is shown in Fig. 14. When the sky is clear, there is no disturbance in the power output. But when the clouds appear, the solar insolation begins to change and the peak power is adjusted by the algorithm according to the new solar insolation level. Fig. 15 shows the LCD readout.

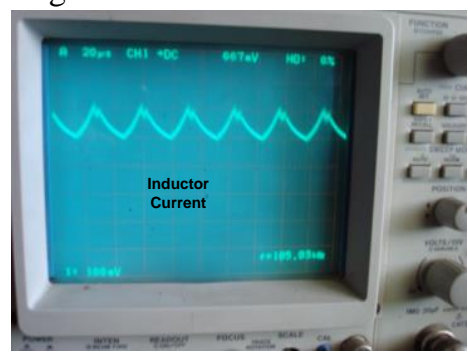

Fig. 13 Photograph of inductor current wave shape

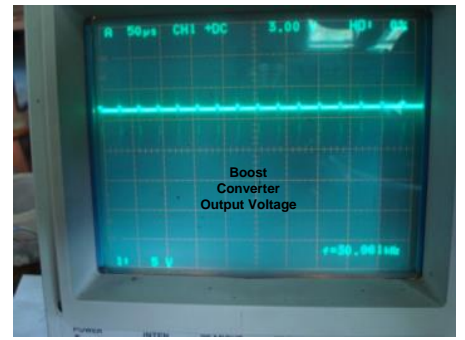

Fig. 14 Photograph of boost converter output voltage

The MPPT efficiency was calculated by manually varying the duty cycle till peak power is tracked. The input voltage, input current, output voltage and output current were measured under various duty cycle ratios and their relationship is plotted in Fig. 16. The plot in Fig. 16 shows that the maximum output power occurs at duty cycle, $\mathrm{D}=$ $44 \%$. The curve shape also shows that the output power decreases on either end of the graph, i.e. at low and high duty cycle ratio. The use of the MPPT enhances the output power by $20 \%$.

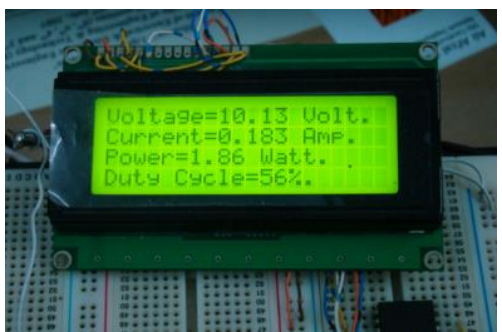

Fig. 15 LCD readout (at running)

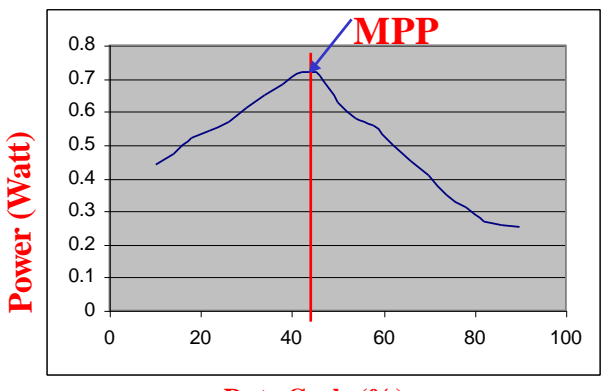

Duty Cycle (\%)

Fig. 16 Duty cycle versus output power

\section{CONCLUSION}

The photovoltaic cell characteristics were simulated and validated in MATLAB. The simulation showed the effects of irradiance and temperature on the operating condition of the photovoltaic array. The simulation results also showed that an increase in irradiance generally causes an increase in the module's output current while an increase in operating temperature generally causes a drop in the module's terminal voltage. An MPPT design was implemented. Experimental results showed that the MMPT has the capability to track the MPP and, the use of the MPPT enhances the output power by $20 \%$. This results in improved efficiency for the operation of renewable energy generation systems. The improved efficiency should lead to significant cost savings in the long run.

\section{REFERENCES}

[1] V. K. Sharma, A. Colangelo and G. Spagna, "Photovoltaic technology: basic concepts, sizing of a standalone photovoltaic system for domestic applications and preliminary economic analysis," Energy Conversion and Management, Elsevier Science, vol. 36, no. 3, pp. 161-174, 1995.

[2] J. L. Santos, F. Antunes, A. Chehab and C. Cruz, "A maximum power point tracker for PV systems using a high performance boost converter," Solar Energy, Elsevier Science, vol. 80, pp. 772-778, 2006.

[3] P. Midya, P. T. Krein, R. J. Turnbull, R. Reppa, and J. Kimball, "Dynamic maximum power point tracker for photovoltaic applications," 27 $7^{\text {th }}$ Annual IEEE Power Electronics Specialists Conference, vol. 2, pp. 1710-1716, 23-27 June 1996.

[4] M. G. Jaboori, M. M. Saied, and A. A. R. Hanafy, "A contribution to the simulation and design optimization of photovoltaic systems," IEEE Transactions on Energy Conversion, vol. 6, no. 3, pp. 401-406, 1991.

[5] B. K. Bose, P. M. Szczesny, and R. L. Steigerwald, "Microcomputer control of a residential photovoltaic power conditioning system," IEEE Transactions on Industrial Applications vol. 21, no. 5, pp. 1182-1191, 1985.

[6] G. Vachtsevanos and K. Kalaitzakiis, "A hybrid photovoltaic simulator for utility interactive studies," IEEE Transactions on Energy Conversion, vol. 2, no. 2, pp. 227-231, 1987. 